\title{
Pädiatrische Gastroenterologie
}

Bauchschmerzen

Übelkeit und

Diarrhoe?

Schuld könnten

\section{Gallensteine schon im Kleinkindalter?}

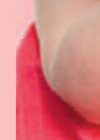

\begin{abstract}
Es klingt unglaublich, doch schon Kinder und Kleinkinder, ja sogar Säuglinge können Gallensteine haben. Anders als beim Erwachsenen handelt es sich dabei meist um Bilirubin- oder Pigmentsteine, und auch die Symptome sind oft grundverschieden. Darüber hinaus muss bei der Therapie anders vorgegangen werden, hieß es beim Pädiater-Forum Gastroenterologie 2011 in München.
\end{abstract}

Bei starken, kolikartigen Bauchschmerzen an Gallensteine zu denken, ist nicht sonderlich schwer. Doch so einfach machen es Kinder ihrem Arzt meist nicht. Gallensteine können sich bei ihnen laut Prof. Dr. Thomas Lang aus Starnberg hingegen mit einem breit gefächerten Symptomspektrum bemerkbar machen. Dazu gehören Unruhe, Übelkeit, Erbrechen und Diarrhoe sowie oft auch Bauchschmerzen, die bis in den Rücken ausstrahlen, aber nicht unbedingt als Kolik beschrieben werden.

Gallensteine sind mit einer Prävalenz von $0,02 \%$ bei Säuglingen bis $0,26 \%$ bei Jugendlichen insgesamt seltene Störungen. In 70\% der Fälle liegen Bilirubinsteine vor, bei 25\% Cholesterinsteine und bei den übrigen Kindern gemischte Steine. Bei der Mehrzahl der Kinder bleiben die Gallensteine asymptomatisch, betonte Lang bei der Veranstal-

\begin{tabular}{l} 
Besuchen Sie das Cgastro \\
Falk Gastro Forum \\
Update Gastro- \\
enterologie \& Hepatologie 2011. \\
Es findet am Samstag, den \\
08. Oktober 2011, in Erlangen statt. \\
Infos: www.drfalkpharma.de/ \\
veranstaltungen \\
\hline
\end{tabular}

tung der Falk Foundation e.V. „Dann sollte man mit der Behandlung zurückhaltend sein“.

\section{Primär konservativ behandeln}

Bestehen jedoch Beschwerden, so ist zunächst eine konservative Therapie indiziert. Bei Bilirubinsteinen, die in aller Regel durch eine hämolytische Erkrankung mit vermehrtem Anfall von unkonjugiertem Bilirubin bedingt sind, geht es primär darum, auslösende Faktoren möglichst zu vermeiden. Liegen Cholesterinsteine oder gemischte Steine vor, so sollte versucht werden, diese durch Gabe der Gallensäure Urso- deoxycholsäure aufzulösen. Erfolgversprechend ist die Behandlung vor allem bei Gallenblasensludge und kleinen Konkrementen. „Rund 60\% der Kinder werden durch die medikamentöse Lyse innerhalb von sechs Monaten steinfrei“, so Lang.

Zurückhaltend ist dagegen die Indikation für eine extrakorporale Stoßwellenlithotripsie zu stellen. Sinn macht diese nur bei solitären, nicht-röntgendichten Konkrementen und zudem auch nur bei größeren Kindern, da ansonsten Koliken durch abgehende Steinpartikel zu befürchten sind. Außerdem ist das Rezidivrisiko mit $80 \%$ hoch, wenngleich es sich durch eine parallele Gabe der Ursodeoxycholsäure senken lässt. Ist eine operative Entfernung der Gallensteine bei Kindern indiziert, so kann dies in 93\% der Fälle laparoskopisch geschehen. Nur in 3-7\% der Fälle ist sekundär eine konventionelle Cholezystektomie erforderlich.

(Christine Vetter)

\section{Auch an Autoimmunprozesse denken}

Noch deutlich unter-diagnostiziert sind bei Kindern und Jugendlichen Autoimmunerkrankungen im Gastrointestinalbereich. Solche Störungen sind zwar selten, sollten nach Prof. Dr. Michael Melter, Regensburg, aber durchaus differentialdiagnostisch in Betracht gezogen werden. Als Beispiel nannte der Mediziner beim Pädiater-Forum Gastroenterologie 2011 die Autoimmunhepatitis (AlH) sowie die primär sklerosierende Cholangitis (PSC) und die autoimmun sklerosierende Cholangitis (ASC), die durch eine Immunreaktion sowohl des Leberparenchyms, als auch der Gallengänge charakterisiert ist.

Daneben sind nach Melter weitere Störungen wie eine De-novo-post-transplantAlH sowie die postinfantile Riesenzellhepatitis mit Coombs-positiver Anämie und/ oder eventuell zusätzlicher Autoimmunthrombozytopenie möglich. Zu bedenken dabei ist, dass die genannten Störungen oft nicht isoliert, sondern assoziiert mit anderen Autoimmunerkrankungen wie einem systemischen Lupus erythematodes oder einer chronisch entzündlichen Darmerkrankung auftreten. „Auch die Zöliakie-assoziierte Hepatopathie ist meines Erachtens den autoimmunen Lebererkrankungen zuzuordnen", betonte der Mediziner in München. 